University of Nebraska - Lincoln

DigitalCommons@University of Nebraska - Lincoln

Winter 10-18-2021

\title{
Role of Library Professionals in Information Dissemination during National Lockdown: A Case Study in Reference to Recent Pandemic
}

Subhajit Panda

Chandigarh University, Mohali, Punjab, suvapanda007@gmail.com

Renuka Sharma

Chandigarh University, Mohali, Punjab

Follow this and additional works at: https://digitalcommons.unl.edu/libphilprac

Part of the Collection Development and Management Commons

Panda, Subhajit and Sharma, Renuka, "Role of Library Professionals in Information Dissemination during National Lockdown: A Case Study in Reference to Recent Pandemic" (2021). Library Philosophy and Practice (e-journal). 6479.

https://digitalcommons.unl.edu/libphilprac/6479 


\title{
Role of Library Professionals in Information Dissemination during National Lockdown: A Case Study in Reference to Recent Pandemic
}

\author{
Mr. Subhajit Panda \\ Assistant Librarian, Chandigarh University, Mohali, Punjab (India) \\ E-mail: suvapanda007@gmail.com \\ Mrs. Renuka Sharma \\ Sr. Assistant Librarian, Chandigarh University, Mohali, Punjab (India) \\ Email: renuka.e1009@cumail.in
}

\begin{abstract}
Libraries play an important role in the everyday life of students and faculty. The COVID-19 pandemic has greatly affected higher education, including libraries systems \& services. This global pandemic almost changes the entire working style of the library from traditional to online. The purpose of this study was to explore the response of the Libraries and LIS professionals during the COVID -19 pandemic and determine their working practices, services practices, and strategies applied and role-played. Libraries see their individual and collective roles of societal and educational dimensions during these uncertain times. The study also made recommendations for the betterment of Libraries. In this study, an online survey was performed using a Google Form questionnaire and send to prominent LIS professionals countrywide. The study covers almost 59 unique libraries from different colleges, universities, public sectors \& information service centres. Out of the collected data, 101 respondents were selected as the final sample of the study. The findings of the study revealed that, among the respondent, $95 \%$ (96) demanded that their library provide online library services, 99\% (100) respondents were positive about their library to provide online ask a librarian facility, and 76\% (77) ensured that their library conducts such QIPs for their staffs during the national lockdown. Further, a total of 73 respondents identified in providing a Remote Access Facility to their library users during the lockdown period. The study concluded that in this new information era library staff, ought to consistently be prepared to confront difficult circumstances. Most libraries recommended in the near future to adopt webinars services and focused on motivating, encouraging the librarians to enhance their digital skills to be in pace with the present digital age.
\end{abstract}

Keywords: COVID -19, Nationwide Lockdown, Academic Libraries, Public Libraries, Technological facilities, Library Professionals.

\section{INTRODUCTION}

India has been hit by a massive second coronavirus wave that commenced in the first week of March 2021 and has continued to grow in size with a large number of cases in subsequent months. The virus was initially discovered on December 31, 2019, in Wuhan, Hubei Province, China (WHO, 2020a), and the first case in India was reported on January 27, 2020, in Kerala (Andrews et al., 2020). According to the recommendation of WHO (2020b) about the social distancing, lockdown, and isolation or quarantine to fight the pandemic, the Indian Government ordered a nationwide lockdown on March 24, 2020 (PIB, 2020). The COVID-19 pandemic has 
altered every part of our life in a somewhat instant manner, leaving little time to adjust and take necessary precautions. As all universities and colleges are closed due to national lockdown, the teaching-learning process and research activities have been badly disrupted. While it is critical to follow the Government's steps to prevent the spread of COVID-19, it is equally important to effectively continue the educational process, making efficient use of technology and other relevant resources. Although the future may be full of uncertainties, challenging times necessitate swift and appropriate decisions (UGC, 2020). This drastic change in Indian Education System changed the need, importance, kind, and way of information dissemination. It also dramatically changes the function of librarians as reliable and consistent disseminators of information and knowledge. In this unique and urgent situation, the role of library professionals is very crucial to make their users aware about the facilities and services provided by them (Dadhe \& Dubey, 2020). The present study aims to determine the varied role of a LIS professionals as an information disseminator by analyse the actual perception of LIS professionals throughout the India.

\section{STUDY OBJECTIVES}

The primary objectives of the study is,

1) To identify whether libraries \& library professionals are providing services to their patrons during lockdown or not

2) To examine the types of services provided by the library professionals during lockdown period

3) To calculate the number of reference queries arrived per day $\&$ average response time

4) To find out the tools used by library professionals in disseminating information during lockdown

5) To identify the level of training and development programme for the library professionals

6) To evaluate the efficiency of staff \& structural availability of the Indian libraries in disseminating information through online mode

\section{LITERATURE REVIEW}

As the pandemic progressed worldwide, several opinions, studies, and blog posts were composed on the subject, focusing on recommendations for library work in this catastrophe. In response to this pandemic, researches, software solutions, risk management guidelines, best practices, and software solutions have also been used \& followed by library associations. Following are some valuable researches which can creates a primary base to understand the need \& importance of the current research.

Ishtiaq et al. (2020) have investigated the role of university libraries in pandemic situation and highlighted how university libraries are contributing to their members for disseminating and mobilizing information during this emergency. The study sample covered 29 academic libraries from private universities in Sindh Province, Pakistan; and the collected data analysed by SPSS software (v. 23.0). The findings of the study revealed that almost $79.3 \%$ libraries providing online library services during the lockdown in contrast to few libraries relying on traditional approach. Rafiq et al. (2021) explored the response of the university libraries' during the COVID-19 pandemic and determined their working practices, services patterns, strategies applied, and role played. An in-depth interview as arranged to collect data from the heads of the seven selected sample university libraries and thematically analysed the collected data. 
Though, in the present situation most of the libraries closed physically access of the library for the patrons, librarians felt that digital divide, lack of digital literacy skills, slow internet speed and less use of library's online resources \& web portals were the major barriers in their transition. The research of Dadhe \& Dubey (2020) purposed to find out how technology became a saviour for the IITs during this pandemic period and explored the type of services provided by IIT libraries based on the contents published on their webpages. Additionally, the study also highlighted the significant initiatives taken by the libraries which can be followed by others to meet the post lockdown needs. Connell et al. (2021) compared the use of library resources \& services (including interlibrary loan, website and discovery tool page views, database use, patron interactions, etc.) at three university libraries before and after the pandemic. The latter part of the 2019 and 2020 spring semesters are the timeframes of focus, although two control timeframes from earlier in those semesters are used to determine how the semesters differed when the coronavirus was not a factor. Brown (2020) stated that the USA libraries have closed their physical buildings and opened their digital doors. Moreover, the American Library Association (ALA) has cancelled their annual conference for the first time in 75 years. Fasae et al., (2020) investigated the academic libraries' response to the pandemic situation in Nigeria. The research was designed upon a structured questionnaire. All total, 584 approved academic institutions in Nigeria were taken as a study sample, among which 108 academic libraries responded to the survey. The finding revealed that nearly all the students are not on campus since they have been directed to vacate their campus as a result of the lockdown. Balachandar and Ramesh (2019) studied how ICT has transformed libraries from book storage facilities to academic data dispersing centres. IFLA (2020) also compiled information \& e-resources regarding the recent situation of libraries around the world and to combat against the pandemic. These sources updated regularly and available its website with free to access facility.

\section{METHODOLOGY}

As the success of a research, depend upon the selection of methodology to conduct the research process. The following methods were adopted for complete the study.

1) Structure survey questionnaire through Google Form: The questionnaire for this shortterm pilot study was designed in Google Form (https://forms.gle/o4JqhsE8hK4HJBQw9) and send to eminent LIS professionals via e-mail. This tool is considered better than the administration due to the lockdown.

2) Sampling Technique: Stratified sampling technique was used in this study to identify the perception of target population. It took almost three months (February 2021 to April 2021) and distributed among 200 (almost) targeted sample groups of which 118 (59\%) took part in this survey from a different region of India.

3) Check Authentication: The data was collected by exporting the Google Sheet as .csv file. After collecting data if any response seems to be odd in nature, the authenticity of the response is examine through their institution website, library website and other external sources.

4) Data Cleansing: Depending on their focus area, authenticity \& completeness, some responses are removed from the final sample. In the present study, among 118 responses $101(85.6 \%)$ was selected as valid response and was imported as a .csv file for further analysis and visualization using spreadsheet software. 
Table 1: Selection of Sample Responses

\begin{tabular}{|c|c|c|}
\hline $\begin{array}{l}\text { Questionnaire } \\
\text { Send }\end{array}$ & Response Received & Valid Response \\
\hline 200 & $\begin{array}{l}118 \\
(59 \% \text { of Total Questionnaire } \\
\text { Send) }\end{array}$ & $\begin{array}{l}101 \\
(85.6 \% \text { of Total Response } \\
\text { Received) } \\
(50.5 \% \text { of Total Questionnaire } \\
\text { Send) }\end{array}$ \\
\hline
\end{tabular}

\section{DATA ANALYSIS AND INTERPRETATION}

Data analysis and interpretation hold a crucial position in the research process. After the selection of the final data sample, the data analysis for the present research was done quantitatively with the help of both descriptive statistics and inferential statistics. It aims to organize, classify and summarize the data being collected for better comprehension and interpretation leading to understand and explore answers or solutions to the research problem which originally triggered the research. The final study sample was categorized according to different parameters and distributed over VR (valid response) percentage and RR (received response) percentage as follows (Table 2-5);

\section{Gender Wise Distribution:}

Table 2 displays the gender-wise distribution of the sample data. It indicates that out of the 101 valid samples, 61.39\% (62) are Males and 34.65\% (35) are females. Again, in terms of RR percentage, Male respondents' covers $52.54 \%$, almost doubled than the female respondents (29.66\%). In addition, there are no respondents from "other" categories (e.g., transgender), while three (3) respondents don't prefer to disclose their names.

Table 2: Gender-wise distribution of sample respondents

\begin{tabular}{|l|r|r|r|}
\hline Gender & $\begin{array}{l}\text { Sample } \\
\text { Size }\end{array}$ & VR Percentage & RR Percentage \\
\hline Male & 62 & 61.39 & 52.54 \\
\hline Female & 35 & 34.65 & 29.66 \\
\hline Other & 0 & 0.00 & 0.00 \\
\hline Prefer not to say & 3 & 2.97 & 2.54 \\
\hline Total & $\mathbf{1 0 1}$ & $\mathbf{1 0 0 . 0 0}$ & $\mathbf{8 5 . 5 9}$ \\
\hline
\end{tabular}

\section{Distribution According to Educational Qualification:}

The educational qualification of the respondent is also considered an important criterion to judge the authenticity of a particular response. Following Table 3 divided into 5 common categories in the LIS education field while considered the addition of the "others" field also. Among the 101 sample respondents, 50.50\%VR (51) are NET Qualified with maximum coverage $(43.22 \% R R)$. It is followed by 25 respondents who completed their $\mathrm{PhD}$. $(24.75 \% \mathrm{VR}$ or $21.19 \%_{R R}$ ). 
Table 3: Distribution according to educational qualification

\begin{tabular}{|l|r|r|r|}
\hline $\begin{array}{l}\text { Educational } \\
\text { Qualification }\end{array}$ & Sample Size & VR Percentage & RR Percentage \\
\hline B.Lib.I.Sc. & 5 & 4.95 & 4.24 \\
\hline M.Lib.I.Sc. & 14 & 13.86 & 11.86 \\
\hline NET Qualified & 51 & 50.50 & 43.22 \\
\hline PhD. & 25 & 24.75 & 21.19 \\
\hline M.Phil. & 3 & 2.97 & 2.54 \\
\hline Others & 3 & 2.97 & 2.54 \\
\hline Total & $\mathbf{1 0 1}$ & $\mathbf{1 0 0 . 0 0}$ & $\mathbf{8 5 . 5 9}$ \\
\hline
\end{tabular}

\section{Designation Wise Distribution:}

Table 4 indicates the designation of the respondents consists of a maximum number of College Librarians (40 or $39.60 \% V R$ or $33.90 \%$ RR). It is followed by 23 Assistant librarians and 18 Library Assistants. Among others, there are 3 University Librarians, 4 Public Librarians, and 2 Deputy Librarians also.

Table 4: Designation-wise distribution of sample respondents

\begin{tabular}{|l|r|r|r|}
\hline Designation & $\begin{array}{r}\text { Sample } \\
\text { Size }\end{array}$ & VR Percentage & RR Percentage \\
\hline University Librarian & 3 & 2.97 & 2.54 \\
\hline Public Librarian & 4 & 3.96 & 3.39 \\
\hline Deputy Librarian & 2 & 1.98 & 1.69 \\
\hline Assistant Librarian & 23 & 22.77 & 19.49 \\
\hline College Librarian & 40 & 39.60 & 33.90 \\
\hline Library Assistant & 18 & 17.82 & 15.25 \\
\hline Other & 11 & 10.89 & 9.32 \\
\hline Total & $\mathbf{1 0 1}$ & $\mathbf{1 0 0 . 0 0}$ & $\mathbf{8 5 . 5 9}$ \\
\hline
\end{tabular}

\section{Affiliation Wise Distribution:}

Out of the 101 respondents, 40 belong to a college in institution affiliation, while 44 others from Universities and 12 from Public Libraries. The rest of the five (5) respondents belongs to some Special Libraries and Information centers. Table 5 below shows the affiliation-wise percentage from both VR \& RR considerations.

Table 5: Affiliation-wise distribution of sample respondents

\begin{tabular}{|l|r|r|r|}
\hline Affiliation & $\begin{array}{r}\text { Sample } \\
\text { Size }\end{array}$ & VR Percentage & RR Percentage \\
\hline College & 40 & 39.60 & 33.90 \\
\hline University & 44 & 43.56 & 37.29 \\
\hline Public Library & 12 & 11.88 & 10.17 \\
\hline Other & 5 & 4.95 & 4.24 \\
\hline
\end{tabular}




\begin{tabular}{|l|r|r|r|} 
Total & 101 & 100.00 & 85.59 \\
\hline
\end{tabular}

\section{RESULT AND DISCUSSION:}

SQ 1: Do your library provides online library services to patrons during Lockdown?

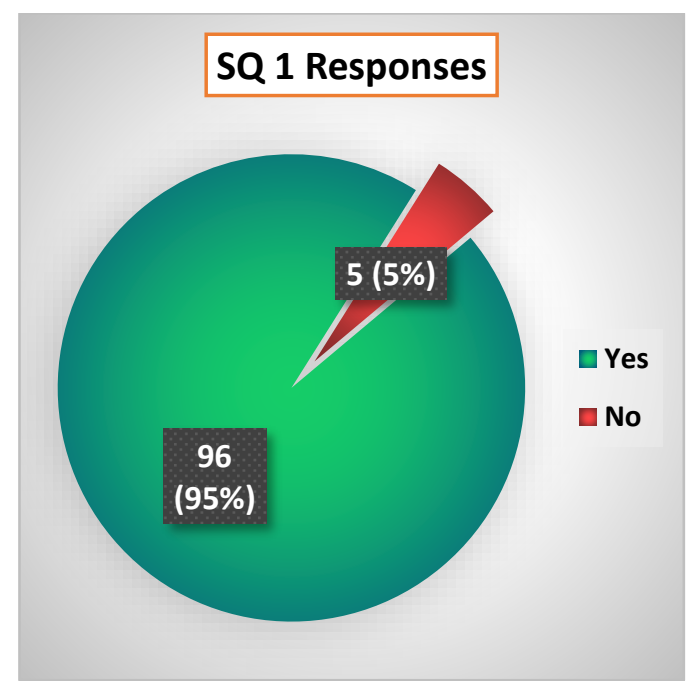

Figure 1: Libraries that provides online library services during lockdown

SQ 1 deals with whether the libraries of the sample respondents provide online library services to their patrons during a lockdown or not. In the questionnaire, it is a simple Yes-No type question, which leads to figure 1 that displays $95 \%$ (96) respondents' library provides online library services during the lockdown while 5 (5\%) others denied declaring so. After further analysis of every single questionnaire, it can be observed from Table 6 that, among the five respondents who claim that their library doesn't provide any types of services during the lockdown, one (1) is a college librarian and four (4) from "others" designation.

Table 6: Designation-wise respondents' category to SQ 1

\begin{tabular}{|l|c|c|c|}
\hline Designation & Yes & No & Total \\
\hline $\begin{array}{l}\text { University } \\
\text { Librarian }\end{array}$ & $\begin{array}{c}3 \\
(3 \%)\end{array}$ & $\begin{array}{c}0 \\
(0 \%)\end{array}$ & $\begin{array}{c}3 \\
(3 \%)\end{array}$ \\
\hline Public Librarian & $\begin{array}{c}4 \\
(4 \%)\end{array}$ & $\begin{array}{c}0 \\
(0 \%)\end{array}$ & $\begin{array}{c}4 \\
(4 \%)\end{array}$ \\
\hline Deputy Librarian & $\begin{array}{c}2 \\
(2 \%)\end{array}$ & $\begin{array}{c}0 \\
(0 \%)\end{array}$ & $\begin{array}{c}2 \\
(2 \%)\end{array}$ \\
\hline Assistant Librarian & $\begin{array}{c}23 \\
(23 \%)\end{array}$ & $\begin{array}{c}2 \% \\
(0 \%)\end{array}$ & $\begin{array}{c}23 \\
(23 \%)\end{array}$ \\
\hline College Librarian & $\begin{array}{c}39 \\
(38 \%)\end{array}$ & $\begin{array}{c}1 \\
(1 \%)\end{array}$ & $\begin{array}{c}401 \\
(39 \%)\end{array}$ \\
\hline Library Assistant & $\begin{array}{c}18 \\
(18 \%)\end{array}$ & $\begin{array}{c}0 \\
(0 \%)\end{array}$ & $\begin{array}{c}18 \\
(18 \%)\end{array}$ \\
\hline Other & $\begin{array}{c}7 \\
(7 \%)\end{array}$ & $\begin{array}{c}4 \\
(4 \%)\end{array}$ & $\begin{array}{c}11 \\
(11 \%)\end{array}$ \\
\hline Total & $\begin{array}{c}\mathbf{9 6} \\
\mathbf{9 5 \%})\end{array}$ & $\begin{array}{c}\mathbf{5} \\
\mathbf{5 \%}\end{array}$ & $\begin{array}{c}\mathbf{1 0 1} \\
(\mathbf{1 0 0 \%})\end{array}$ \\
\hline
\end{tabular}




\section{SQ 2: Which type of services provided by your Library for Patrons in Lockdown?}

Among the 96 positive respondents ( $N$ for $S Q 2$ ) from SQ 1 about providing online library services during the lockdown period, SQ 2 categorizes the possible types of popular services that could be provided to the users (see table 7). Out of the 96 respondents, E-mail Management is the most commonly provided service with a coverage of $100 \%$ (96), and Social Sites in exact next (86 or $96 \%)$. It is closely followed by Remote Access Facility and Live Chat services with $76 \%$ (or 73 ) and $75 \%$ (or 72) coverage, respectably. It is also important to note that Mobile Application Services, which is significant for modern age libraries with changing users' needs, are not familiar among the sample respondents' libraries (18\% or 17).

Table 7: Types of service provided by respondents' libraries during lockdown

\begin{tabular}{|c|c|c|c|c|c|c|c|c|}
\hline Types of Services & $\begin{array}{c}\mathbf{U L} \\
\mathbf{N}=\mathbf{3}\end{array}$ & $\begin{array}{c}P L \\
N=4\end{array}$ & $\begin{array}{c}\mathrm{DL} \\
\mathrm{N}=2\end{array}$ & \begin{tabular}{|c|}
$\mathbf{A L}$ \\
$\mathbf{N}=\mathbf{2 3}$ \\
\end{tabular} & $\begin{array}{c}\mathrm{CL} \\
\mathrm{N}=39 \\
\end{array}$ & $\begin{array}{c}\text { LA } \\
\mathrm{N}=18\end{array}$ & $\begin{array}{c}\mathrm{O} \\
\mathrm{N}=7\end{array}$ & $\begin{array}{l}\text { Total } \\
\mathrm{N}=96\end{array}$ \\
\hline Mobile application services & $\begin{array}{c}1 \\
(33 \%) \\
\end{array}$ & $\begin{array}{c}0 \\
(0 \%) \\
\end{array}$ & $\begin{array}{c}1 \\
(50 \%) \\
\end{array}$ & \begin{tabular}{|c|}
8 \\
$(35 \%)$ \\
\end{tabular} & $\begin{array}{c}4 \\
(10 \%) \\
\end{array}$ & $\begin{array}{c}0 \\
(0 \%) \\
\end{array}$ & $\begin{array}{c}3 \\
(43 \%) \\
\end{array}$ & $\begin{array}{c}17 \\
(18 \%) \\
\end{array}$ \\
\hline Live Chat & $\begin{array}{c}3 \\
(100 \% \\
)\end{array}$ & $\begin{array}{c}3 \\
(75 \%)\end{array}$ & $\begin{array}{c}2 \\
(100 \% \\
)\end{array}$ & $\begin{array}{c}19 \\
(83 \%)\end{array}$ & $\begin{array}{c}24 \\
(62 \%)\end{array}$ & $\begin{array}{c}17 \\
(94 \%)\end{array}$ & $\begin{array}{c}4 \\
(57 \%)\end{array}$ & $\begin{array}{c}72 \\
(75 \%)\end{array}$ \\
\hline Social Sites & $\begin{array}{c}3 \\
(100 \% \\
)\end{array}$ & $\begin{array}{c}4 \\
(100 \%)\end{array}$ & $\begin{array}{c}2 \\
(100 \% \\
)\end{array}$ & $\begin{array}{c}19 \\
(83 \%)\end{array}$ & $\begin{array}{c}35 \\
(90 \%)\end{array}$ & $\begin{array}{c}18 \\
(100 \%)\end{array}$ & $\begin{array}{c}7 \\
(100 \%)\end{array}$ & $\begin{array}{c}86 \\
(90 \%)\end{array}$ \\
\hline E-Mail Management & $\begin{array}{c}3 \\
(100 \% \\
\end{array}$ & $\begin{array}{c}4 \\
(100 \%)\end{array}$ & $\begin{array}{c}2 \\
(100 \% \\
)\end{array}$ & $\begin{array}{c}23 \\
(100 \%)\end{array}$ & $\begin{array}{c}39 \\
(100 \%)\end{array}$ & $\begin{array}{c}18 \\
(100 \%)\end{array}$ & $\begin{array}{c}7 \\
(100 \%)\end{array}$ & $\begin{array}{c}96 \\
(100 \% \\
) \\
\end{array}$ \\
\hline Remote Access Facility & $\begin{array}{c}3 \\
(100 \% \\
) \\
\end{array}$ & $\begin{array}{c}1 \\
(25 \%)\end{array}$ & $\begin{array}{c}2 \\
(100 \% \\
)\end{array}$ & $\begin{array}{c}19 \\
(83 \%)\end{array}$ & $\begin{array}{c}32 \\
(82 \%)\end{array}$ & $\begin{array}{c}11 \\
(61 \%)\end{array}$ & $\begin{array}{c}5 \\
(71 \%)\end{array}$ & $\begin{array}{c}73 \\
(76 \%)\end{array}$ \\
\hline Other services & $\begin{array}{c}3 \\
(100 \% \\
\end{array}$ & $\begin{array}{c}4 \\
(100 \%)\end{array}$ & $\begin{array}{c}2 \\
(100 \% \\
)\end{array}$ & $\begin{array}{c}20 \\
(87 \%)\end{array}$ & $\begin{array}{c}17 \\
(44 \%)\end{array}$ & $\begin{array}{c}8 \\
(44 \%)\end{array}$ & $\begin{array}{c}0 \\
(0 \%)\end{array}$ & $\begin{array}{c}54 \\
(56 \%)\end{array}$ \\
\hline
\end{tabular}

\section{SQ 3: Which type of resources you provide through remote access facility?}

From the responses of SQ 2, it can be easily identified that out of the total 96 respondents, 73 responded ( $N$ for $S Q 3$ ) positively about providing a Remote Access Facility to their library users. Now, following Table 8 listed some significant e-resources most commonly used for Remote Access purposes. Amongst them, the DELNET consortium has the majority of the coverage (59 or $81 \%$ ), closely followed by EDS \& WoS, both with $67 \%$ (49) coverage. Surprisingly, the "others" field possesses maximum coverage (63 or $86 \%$ ) above all, concerning the 7 mentioned e-resource databases in Table 8. It indicates that the sample respondents' educational institutions use e-resource databases for remote access facilities as per their particular needs and can't generalize easily.

Table 8: Type of resources provided by respondents' libraries for remote access facility 


\begin{tabular}{|c|c|c|c|c|c|c|c|c|}
\hline $\begin{array}{l}\text { Types of } \\
\text { Resource }\end{array}$ & $\begin{array}{c}\mathrm{UL} \\
\mathbf{N}=\mathbf{3}\end{array}$ & $\begin{array}{c}\mathrm{PL} \\
\mathrm{N}=1\end{array}$ & $\begin{array}{c}\mathrm{DL} \\
\mathrm{N}=2\end{array}$ & $\begin{array}{c}\mathrm{AL} \\
\mathrm{N}=19\end{array}$ & $\begin{array}{c}\mathrm{CL} \\
\mathrm{N}=32\end{array}$ & $\underset{\mathbf{N}=11}{\mathbf{L A}}$ & $\underset{N=5}{O}$ & $\begin{array}{l}\text { Total } \\
\mathrm{N}=73\end{array}$ \\
\hline $\begin{array}{l}\text { EBSCO } \\
\text { Discovery Service }\end{array}$ & $\begin{array}{c}3 \\
(100 \% \\
)\end{array}$ & $\begin{array}{c}1 \\
(100 \% \\
)\end{array}$ & $\begin{array}{c}2 \\
(100 \% \\
)\end{array}$ & $\begin{array}{c}19 \\
(100 \% \\
)\end{array}$ & $\begin{array}{c}11 \\
(34 \%)\end{array}$ & $\begin{array}{c}8 \\
(73 \%)\end{array}$ & $\begin{array}{c}5 \\
(100 \% \\
)\end{array}$ & $\begin{array}{c}49 \\
(67 \%)\end{array}$ \\
\hline Web of Science & $\begin{array}{c}3 \\
(100 \% \\
)\end{array}$ & $\begin{array}{c}1 \\
(100 \% \\
\quad\end{array}$ & $\begin{array}{c}2 \\
(100 \% \\
)\end{array}$ & $\begin{array}{c}19 \\
(100 \% \\
)\end{array}$ & $\begin{array}{c}11 \\
(34 \%)\end{array}$ & $\begin{array}{c}8 \\
(73 \%)\end{array}$ & $\begin{array}{c}5 \\
(100 \% \\
\quad)\end{array}$ & $\begin{array}{c}49 \\
(67 \%)\end{array}$ \\
\hline J-Gate & $\begin{array}{c}3 \\
(100 \% \\
)\end{array}$ & $\begin{array}{c}1 \\
(100 \% \\
\quad\end{array}$ & $\begin{array}{c}2 \\
(100 \% \\
)\end{array}$ & $\begin{array}{c}11 \\
(58 \%)\end{array}$ & $\begin{array}{c}7 \\
(22 \%)\end{array}$ & $\begin{array}{c}6 \\
(55 \%)\end{array}$ & $\begin{array}{c}5 \\
(100 \% \\
\quad\end{array}$ & $\begin{array}{c}35 \\
(48 \%)\end{array}$ \\
\hline Manupatra & $\begin{array}{c}2 \\
(67 \%)\end{array}$ & $\begin{array}{c}0 \\
(0 \%)\end{array}$ & $\begin{array}{c}2 \\
(100 \% \\
)\end{array}$ & $\begin{array}{c}8 \\
(42 \%)\end{array}$ & $\begin{array}{c}3 \\
(9 \%)\end{array}$ & $\begin{array}{c}0 \\
(0 \%)\end{array}$ & $\begin{array}{c}0 \\
(0 \%)\end{array}$ & $\begin{array}{c}15 \\
(21 \%)\end{array}$ \\
\hline Pearson & $\begin{array}{c}3 \\
(100 \% \\
\quad)\end{array}$ & $\begin{array}{c}1 \\
(100 \% \\
)\end{array}$ & $\begin{array}{c}2 \\
(100 \% \\
)\end{array}$ & $\begin{array}{c}18 \\
(95 \%)\end{array}$ & $\begin{array}{c}11 \\
(34 \%)\end{array}$ & $\begin{array}{c}7 \\
(64 \%)\end{array}$ & $\begin{array}{c}3 \\
(60 \%)\end{array}$ & $\begin{array}{c}45 \\
(62 \%)\end{array}$ \\
\hline N-List & $\begin{array}{c}0 \\
(0 \%)\end{array}$ & $\begin{array}{c}0 \\
(0 \%)\end{array}$ & $\begin{array}{c}0 \\
(0 \%)\end{array}$ & $\begin{array}{c}6 \\
(32 \%)\end{array}$ & $\begin{array}{c}32 \\
(100 \% \\
)\end{array}$ & $\begin{array}{c}3 \\
(27 \%)\end{array}$ & $\begin{array}{c}2 \\
(40 \%)\end{array}$ & $\begin{array}{c}43 \\
(59 \%)\end{array}$ \\
\hline DELNET & $\begin{array}{c}2 \\
(67 \%)\end{array}$ & $\begin{array}{c}1 \\
(100 \% \\
)\end{array}$ & $\begin{array}{c}2 \\
(100 \% \\
)\end{array}$ & $\begin{array}{c}17 \\
(89 \%)\end{array}$ & $\begin{array}{c}24 \\
(75 \%)\end{array}$ & $\begin{array}{c}11 \\
(100 \% \\
)\end{array}$ & $\begin{array}{c}2 \\
(40 \%)\end{array}$ & $\begin{array}{c}59 \\
(81 \%)\end{array}$ \\
\hline Other & $\begin{array}{c}3 \\
(100 \% \\
)\end{array}$ & $\begin{array}{c}1 \\
(100 \% \\
)\end{array}$ & $\begin{array}{c}2 \\
(100 \% \\
)\end{array}$ & $\begin{array}{c}19 \\
(100 \% \\
)\end{array}$ & $\begin{array}{c}28 \\
(88 \%)\end{array}$ & $\begin{array}{c}5 \\
(45 \%)\end{array}$ & $\begin{array}{c}5 \\
(100 \% \\
\quad)\end{array}$ & $\begin{array}{c}63 \\
(86 \%)\end{array}$ \\
\hline
\end{tabular}

SQ 4: Do your library provide online ask a librarian facility to resolve queries of your library users during lockdown?

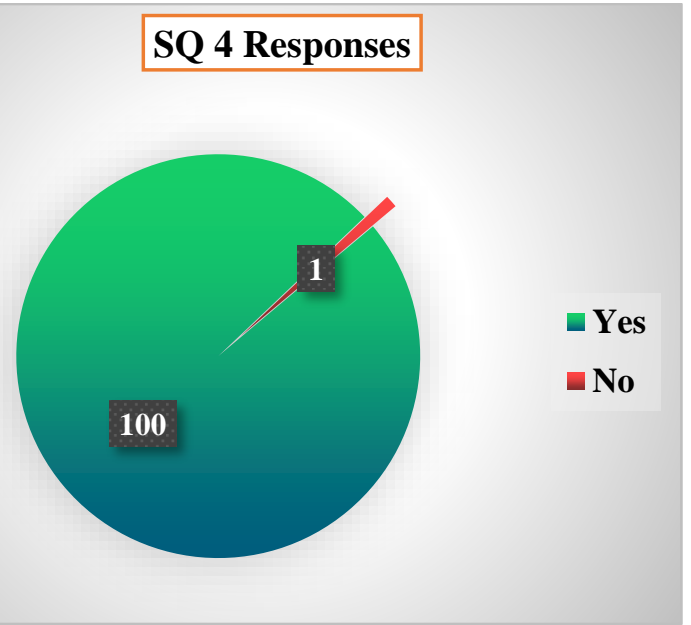

Figure 2: Designation-wise respondents' category to SQ 4 
SQ 4 deals with whether the sample respondents' libraries provide online ask a librarian facility for reference queries to their patrons during a lockdown or not. In the questionnaire, it is a simple Yes-No type question, which leads to figure 2 that displays 99\% (100) respondents' library provides online ask a librarian facility during the lockdown while $1(1 \%)$ others denied declaring so. After further analysis of every single questionnaire, it can be observed from Table 9 that, the only one respondent who claim that their library doesn't provide reference desk or ask a librarian facility during the lockdown is a library assistant from one general in designation.

Table 9: Designation-wise respondents' category to SQ 4

\begin{tabular}{|l|c|c|c|}
\hline Designation & Yes & No & Total \\
\hline University & 3 & 0 & 3 \\
$(3 \%)$ & $(0 \%)$ & $(3 \%)$ \\
\hline Public Librarian & $\begin{array}{c}4 \\
(4 \%)\end{array}$ & $\begin{array}{c}0 \\
(0 \%)\end{array}$ & $\begin{array}{c}4 \\
(4 \%)\end{array}$ \\
\hline Deputy Librarian & $\begin{array}{c}2 \\
(2 \%)\end{array}$ & $\begin{array}{c}0 \\
(0 \%)\end{array}$ & $\begin{array}{c}2 \\
(2 \%)\end{array}$ \\
\hline Assistant Librarian & $\begin{array}{c}23 \\
(23 \%)\end{array}$ & $\begin{array}{c}0 \\
(0 \%)\end{array}$ & $\begin{array}{c}23 \\
(23 \%)\end{array}$ \\
\hline College Librarian & $\begin{array}{c}40 \\
(39 \%)\end{array}$ & $\begin{array}{c}0 \\
(0 \%)\end{array}$ & $\begin{array}{c}40 \\
(39 \%)\end{array}$ \\
\hline Library Assistant & $\begin{array}{c}17 \\
(17 \%)\end{array}$ & $\begin{array}{c}1 \\
(1 \%)\end{array}$ & $\begin{array}{c}18 \\
(18 \%)\end{array}$ \\
\hline Other & $\begin{array}{c}11 \\
(11 \%)\end{array}$ & $\begin{array}{c}0 \\
(0 \%)\end{array}$ & $\begin{array}{c}11 \\
(11 \%)\end{array}$ \\
\hline Total & $\begin{array}{c}\mathbf{1 0 0} \\
(\mathbf{9 9 \%})\end{array}$ & $\begin{array}{c}\mathbf{1} \\
(\mathbf{1 \%})\end{array}$ & $\begin{array}{c}\mathbf{1 0 1} \\
(\mathbf{1 0 0} \%)\end{array}$ \\
\hline
\end{tabular}

\section{SQ 5: Average number of Reference Queries arrived in a day?}

The 100 respondents who claim that their parent library provides online ask a librarian facility for reference queries are further detailed in SQ 5 by distributing them according to received number of responses and users' type. In Table 9, five possible users' type is listed corresponding with the received number of responses. It can be easily identified that $\leq 5$ queries per day is common \& in the majority number for every type of user, which leads to overall coverage of $41.2 \%$ (206) of that type. It is followed by 6-10 \& 11-15 queries per day with $22.0 \%$ (110) \& $15.4 \%$ (77) overall coverage respectively.

Table 9: Average number of reference queries per day per user types

\begin{tabular}{|l|c|c|c|c|c|c|c|}
\hline Users' Type & No Queries & $\mathbf{5}$ & $\mathbf{6 - 1 0}$ & $\mathbf{1 1 - 1 5}$ & $\mathbf{1 6 - 2 0}$ & $\mathbf{> 2 0}$ & Total \\
\hline \multirow{2}{*}{ UG Student } & 2 & 42 & 18 & 24 & 11 & 3 & 100 \\
& $(2 \%)$ & $(42 \%)$ & $(18 \%)$ & $(24 \%)$ & $(11 \%)$ & $(3 \%)$ & $(100 \%)$ \\
\hline \multirow{2}{*}{ PG Student } & 4 & 39 & 22 & 12 & 19 & 4 & 100 \\
& $(4 \%)$ & $(39 \%)$ & $(22 \%)$ & $(12 \%)$ & $(19 \%)$ & $(4 \%)$ & $(100 \%)$ \\
\hline \multirow{2}{*}{ Research scholar } & 17 & 28 & 25 & 8 & 10 & 12 & 100 \\
& $(17 \%)$ & $(28 \%)$ & $(25 \%)$ & $(8 \%)$ & $(10 \%)$ & $(12 \%)$ & $(100 \%)$ \\
\hline
\end{tabular}




\begin{tabular}{|l|c|c|c|c|c|c|c|}
\hline Teaching Faculty & $\begin{array}{c}0 \\
(0 \%)\end{array}$ & $\begin{array}{c}35 \\
(35 \%)\end{array}$ & $\begin{array}{c}26 \\
(26 \%)\end{array}$ & $\begin{array}{c}23 \\
(23 \%)\end{array}$ & $\begin{array}{c}6 \\
(6 \%)\end{array}$ & $\begin{array}{c}10 \\
(10 \%)\end{array}$ & $\begin{array}{c}100 \\
(100 \%)\end{array}$ \\
\hline Non-Teaching & 1 & 62 & 19 & 10 & 2 & 6 & 100 \\
Faculty & $(1 \%)$ & $(62 \%)$ & $(19 \%)$ & $(10 \%)$ & $(2 \%)$ & $(6 \%)$ & $(100 \%)$ \\
\hline Overall Coverage & $\mathbf{2 4}$ & $\mathbf{2 0 6}$ & $\mathbf{1 1 0}$ & $\mathbf{7 7}$ & $\mathbf{4 8}$ & $\mathbf{3 5}$ & $\mathbf{N}=\mathbf{5 0 0}$ \\
\hline Overall Percentage & $\mathbf{4 . 8 \%}$ & $\mathbf{4 1 . 2 \%}$ & $\mathbf{2 2 . 0 \%}$ & $\mathbf{1 5 . 4 \%}$ & $\mathbf{9 . 6 \%}$ & $\mathbf{7 . 0 \%}$ & $\mathbf{1 0 0 . 0 0 \%}$ \\
\hline
\end{tabular}

SQ 6: How is your response time regarding reference queries in your libraries?

Table 10 reflects the responses time taken by the respondents to answer the queries of the users. A majority of $47 \%$ (47) of the 100 positive respondents (from $S Q 4$ ) answer within 1 hour to any query received from the user. Respondents who responded within 5 minutes came in second ( 33 or $33 \%$ ), while respondents responses within 1 day placed exactly next (12 or $12 \%$ ).

Table 10: Response time regarding reference queries in libraries

\begin{tabular}{|c|c|c|c|c|c|c|c|c|}
\hline $\begin{array}{l}\text { Response } \\
\text { Time }\end{array}$ & $\mathbf{U L}$ & PL & DL & $\mathbf{A L}$ & $\mathbf{C L}$ & $\mathbf{L A}$ & $\mathbf{O}$ & $\begin{array}{c}\text { Overall } \\
\text { Coverag } \\
\text { e }\end{array}$ \\
\hline within 5 mins & $\begin{array}{c}2 \\
(67 \%)\end{array}$ & $\begin{array}{c}0 \\
(0 \%)\end{array}$ & $\begin{array}{c}0 \\
(0 \%)\end{array}$ & $\begin{array}{c}11 \\
(48 \%)\end{array}$ & $\begin{array}{c}12 \\
(30 \%)\end{array}$ & $\begin{array}{c}6 \\
(35 \%)\end{array}$ & $\begin{array}{c}2 \\
(18 \%)\end{array}$ & $\begin{array}{c}33 \\
(33 \%)\end{array}$ \\
\hline within $1 \mathrm{hr}$. & $\begin{array}{c}1 \\
(33 \%)\end{array}$ & $\begin{array}{c}2 \\
(50 \%)\end{array}$ & $\begin{array}{c}2 \\
(100 \%)\end{array}$ & $\begin{array}{c}9 \\
(39 \%)\end{array}$ & $\begin{array}{c}24 \\
(60 \%)\end{array}$ & $\begin{array}{c}5 \\
(29 \%)\end{array}$ & $\begin{array}{c}4 \\
(36 \%)\end{array}$ & $\begin{array}{c}47 \\
(47 \%)\end{array}$ \\
\hline within 1 day & $\begin{array}{c}0 \\
(0 \%)\end{array}$ & $\begin{array}{c}2 \\
(50 \%) \\
\end{array}$ & $\begin{array}{c}0 \\
(0 \%)\end{array}$ & $\begin{array}{c}3 \\
(13 \%)\end{array}$ & $\begin{array}{c}1 \\
(3 \%)\end{array}$ & $\begin{array}{c}2 \\
(12 \%)\end{array}$ & $\begin{array}{c}4 \\
(36 \%)\end{array}$ & $\begin{array}{c}12 \\
(12 \%)\end{array}$ \\
\hline $\begin{array}{l}\text { more than } 1 \\
\text { day }\end{array}$ & $\begin{array}{c}0 \\
(0 \%)\end{array}$ & $\begin{array}{c}0 \\
(0 \%)\end{array}$ & $\begin{array}{c}0 \\
(0 \%)\end{array}$ & $\begin{array}{c}0 \\
(0 \%)\end{array}$ & $\begin{array}{c}2 \\
(5 \%)\end{array}$ & $\begin{array}{c}4 \\
(24 \%)\end{array}$ & $\begin{array}{c}0 \\
(0 \%)\end{array}$ & $\begin{array}{c}6 \\
(6 \%)\end{array}$ \\
\hline $\begin{array}{l}\text { not response } \\
\text { all time }\end{array}$ & $\begin{array}{c}0 \\
(0 \%)\end{array}$ & $\begin{array}{c}0 \\
(0 \%)\end{array}$ & $\begin{array}{c}0 \\
(0 \%)\end{array}$ & $\begin{array}{c}0 \\
(0 \%)\end{array}$ & $\begin{array}{c}1 \\
(3 \%)\end{array}$ & $\begin{array}{c}0 \\
(0 \%)\end{array}$ & $\begin{array}{c}1 \\
(9 \%)\end{array}$ & $\begin{array}{c}2 \\
(2 \%)\end{array}$ \\
\hline $\begin{array}{l}\text { Total } \\
\text { (individual) }\end{array}$ & $\begin{array}{c}3 \\
(100 \%)\end{array}$ & $\begin{array}{c}4 \\
(100 \%)\end{array}$ & $\begin{array}{c}2 \\
(100 \%)\end{array}$ & $\begin{array}{c}23 \\
(100 \%)\end{array}$ & $\begin{array}{c}40 \\
(100 \%)\end{array}$ & $\begin{array}{c}17 \\
(100 \%)\end{array}$ & $\begin{array}{c}11 \\
(100 \%)\end{array}$ & $\begin{array}{c}\mathrm{N}=100 \\
(100 \%)\end{array}$ \\
\hline
\end{tabular}

\section{SQ 7: Web tools used by your library to respond user's query...}

Table 11 deals with the type of reference tools used by the respondents' library to answer the reference query of the users. According to the table, out of the five mentioned web tools Gmail or Blue-mail services and Telephonic interaction used by $100 \%$ (100) of the respondent. Instant messaging services are close behind, with 95\% (95) coverage. In addition to these five professions, $13 \%$ (13) of respondents chose other fields.

Table 11: Web tools used to respond reference queries

\begin{tabular}{|c|c|c|c|c|c|c|c|c|}
\hline $\begin{array}{l}\text { Type of } \\
\text { Reference Tools }\end{array}$ & $\begin{array}{c}\mathbf{U L} \\
(\mathbf{N}=\mathbf{3})\end{array}$ & $\begin{array}{c}\text { PL } \\
(\mathrm{N}=4)\end{array}$ & $\begin{array}{c}\mathrm{DL} \\
(\mathrm{N}=2)\end{array}$ & $\begin{array}{c}A L \\
(\mathrm{~N}=23 \\
)\end{array}$ & $\begin{array}{c}C L \\
(\mathrm{~N}=40 \\
\quad)\end{array}$ & $\begin{array}{c}\text { LA } \\
(\mathbf{N}=\mathbf{1 7})\end{array}$ & $\begin{array}{c}\mathbf{O} \\
(\mathbf{N}=\mathbf{1 1})\end{array}$ & $\begin{array}{c}\text { Overall } \\
\text { Coverag } \\
\mathbf{e} \\
(\mathrm{N}=100)\end{array}$ \\
\hline $\begin{array}{l}\text { Gmail/Blue-mail } \\
\text { for Mailing } \\
\text { Services }\end{array}$ & $\begin{array}{c}3 \\
(100 \% \\
)\end{array}$ & $\begin{array}{c}4 \\
(100 \% \\
)\end{array}$ & $\begin{array}{c}2 \\
(100 \% \\
)\end{array}$ & $\begin{array}{c}23 \\
(100 \% \\
)\end{array}$ & $\begin{array}{c}40 \\
(100 \% \\
)\end{array}$ & $\begin{array}{c}17 \\
(100 \%)\end{array}$ & $\begin{array}{c}11 \\
(100 \%)\end{array}$ & $\begin{array}{c}100 \\
(100 \%)\end{array}$ \\
\hline
\end{tabular}




\begin{tabular}{|c|c|c|c|c|c|c|c|c|}
\hline $\begin{array}{l}\text { Chatbot for Live } \\
\text { Chat \& Virtual } \\
\text { Assistance }\end{array}$ & $\begin{array}{c}2 \\
(67 \%)\end{array}$ & $\begin{array}{c}1 \\
(25 \%)\end{array}$ & $\begin{array}{c}1 \\
(50 \%)\end{array}$ & $\begin{array}{c}9 \\
(39 \%)\end{array}$ & $\begin{array}{c}2 \\
(5 \%)\end{array}$ & $\begin{array}{c}0 \\
(0 \%)\end{array}$ & $\begin{array}{c}0 \\
(0 \%)\end{array}$ & $\begin{array}{c}15 \\
(15 \%)\end{array}$ \\
\hline $\begin{array}{l}\text { Instant } \\
\text { Messaging } \\
\text { Services through } \\
\text { Mobile }\end{array}$ & $\begin{array}{c}3 \\
(100 \% \\
\quad)\end{array}$ & $\begin{array}{c}4 \\
(100 \% \\
\quad)\end{array}$ & $\begin{array}{c}2 \\
(100 \% \\
\quad)\end{array}$ & $\begin{array}{c}23 \\
(100 \% \\
\quad)\end{array}$ & $\begin{array}{c}35 \\
(88 \%)\end{array}$ & $\begin{array}{c}17 \\
(100 \%)\end{array}$ & $\begin{array}{c}11 \\
(100 \%)\end{array}$ & $\begin{array}{c}95 \\
(95 \%)\end{array}$ \\
\hline $\begin{array}{l}\text { Social media for } \\
\text { quick \& easy } \\
\text { dissemination }\end{array}$ & $\begin{array}{c}2 \\
(67 \%)\end{array}$ & $\begin{array}{c}3 \\
(75 \%)\end{array}$ & $\begin{array}{c}2 \\
(100 \% \\
)\end{array}$ & $\begin{array}{c}14 \\
(61 \%)\end{array}$ & $\begin{array}{c}27 \\
(68 \%)\end{array}$ & $\begin{array}{c}5 \\
(29 \%)\end{array}$ & $\begin{array}{c}3 \\
(27 \%)\end{array}$ & $\begin{array}{c}56 \\
(56 \%)\end{array}$ \\
\hline $\begin{array}{l}\text { Telephonic } \\
\text { interaction for } \\
\text { clear } \\
\text { understanding }\end{array}$ & $\begin{array}{c}3 \\
(100 \% \\
\quad)\end{array}$ & $\begin{array}{c}4 \\
(100 \% \\
)\end{array}$ & $\begin{array}{c}2 \\
(100 \% \\
)\end{array}$ & $\begin{array}{c}23 \\
(100 \% \\
)\end{array}$ & $\begin{array}{c}40 \\
(100 \% \\
)\end{array}$ & $\begin{array}{c}17 \\
(100 \%)\end{array}$ & $\begin{array}{c}11 \\
(100 \%)\end{array}$ & $\begin{array}{c}100 \\
(100 \%)\end{array}$ \\
\hline Other medium & $\begin{array}{c}1 \\
(33 \%)\end{array}$ & $\begin{array}{c}1 \\
(25 \%)\end{array}$ & $\begin{array}{c}0 \\
(0 \%)\end{array}$ & $\begin{array}{c}7 \\
(30 \%) \\
\end{array}$ & $\begin{array}{c}4 \\
(10 \%) \\
\end{array}$ & $\begin{array}{c}0 \\
(0 \%) \\
\end{array}$ & $\begin{array}{c}0 \\
(0 \%)\end{array}$ & $\begin{array}{c}13 \\
(13 \%)\end{array}$ \\
\hline
\end{tabular}

SQ 8: Do your Library conduct quality improvement programme for Library Staff?

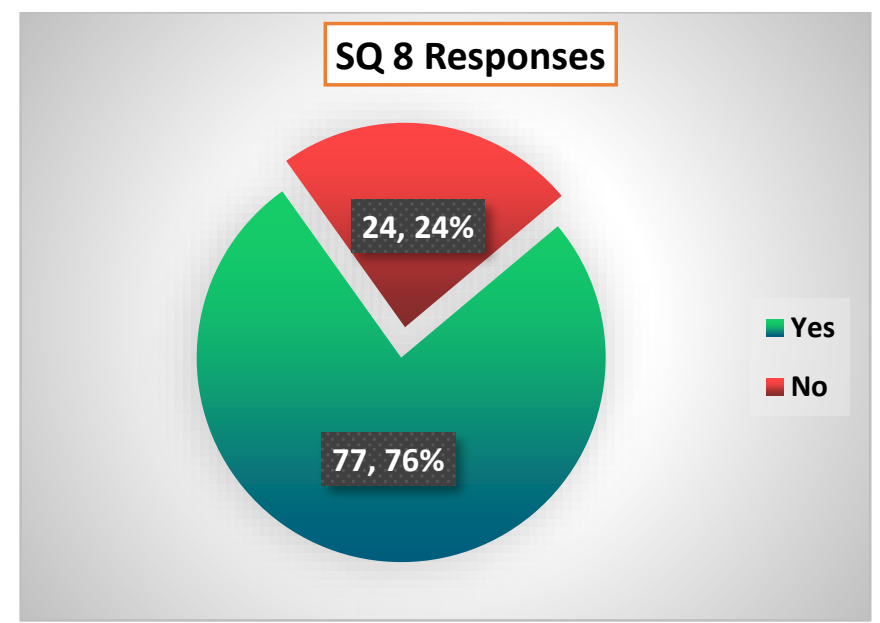

Figure 3: Status of Conduction of QIPs in Libraries

Quality improvement is a continual desire in every service business and QIPs for employees, such as hands-on workshops, seminars, and literacy programmes, are critical in coping with the changes and modifications brought about by quality improvement. Figure 3 shows whether or not the library committees of the respondents organise a QIP for their employees. Among the 101 respondent, 76\% (77) ensured that their library conducts such QIPs for their staffs, while $24(24 \%)$ decline about the same. On further analysis of the responses (see Table 12) reveals that out of the 24 (24\%) declining respondents, 9\% (9) are College Librarian, 6\% (6) are Assistant Librarian, 2\% (2) are Library Assistant and 4\% (4) are from other categories.

Table 12: Status of Conduction of QIPs in Libraries

\begin{tabular}{|l|c|c|c|}
\hline Designation & Yes & No & Total \\
\hline University Librarian & $\begin{array}{c}3 \\
(3 \%)\end{array}$ & $\begin{array}{c}0 \\
(0 \%)\end{array}$ & $\begin{array}{c}3 \\
(3 \%)\end{array}$ \\
\hline Public Librarian & $\begin{array}{c}1 \\
(1 \%)\end{array}$ & $\begin{array}{c}3 \\
(3 \%)\end{array}$ & $\begin{array}{c}4 \\
(4 \%)\end{array}$ \\
\hline
\end{tabular}




\begin{tabular}{|l|c|c|c|}
\hline Deputy Librarian & $\begin{array}{c}2 \\
(2 \%)\end{array}$ & $\begin{array}{c}0 \\
(0 \%)\end{array}$ & $\begin{array}{c}2 \\
(2 \%)\end{array}$ \\
\hline Assistant Librarian & $\begin{array}{c}17 \\
(17 \%)\end{array}$ & $\begin{array}{c}6 \\
(6 \%)\end{array}$ & $\begin{array}{c}23 \\
(23 \%)\end{array}$ \\
\hline College Librarian & $\begin{array}{c}31 \\
(30 \%)\end{array}$ & $\begin{array}{c}9 \\
(9 \%)\end{array}$ & $\begin{array}{c}40 \\
(39 \%)\end{array}$ \\
\hline Library Assistant & $\begin{array}{c}16 \\
(16 \%)\end{array}$ & $\begin{array}{c}2 \\
(2 \%)\end{array}$ & $\begin{array}{c}18 \\
(18 \%)\end{array}$ \\
\hline Other & $\begin{array}{c}7 \\
(7 \%)\end{array}$ & $\begin{array}{c}4 \\
(4 \%)\end{array}$ & $\begin{array}{c}11 \\
(11 \%)\end{array}$ \\
\hline Total & $\begin{array}{c}\mathbf{7 7} \\
\mathbf{( 7 6 \% )}\end{array}$ & $\begin{array}{c}\mathbf{2 4 \%} \\
\mathbf{1 0 1}\end{array}$ & $\mathbf{( 1 0 0 \% )}$ \\
\hline
\end{tabular}

\section{SQ 9: Which type of quality improvement programme (QIP) conduct by your Library?}

In consequence to SQ 10, SQ 9 further analyse the type of QIPs organised by the libraries of the respondents for their overall progress. According to Table 13, all of the 77 respondent libraries conducted Literacy session and inter \& intra - library discussion session as most necessary QIP among all. Conduction of National \& International webinars placed in second position with $94 \%$ (72) responses, followed by hands on workshops with $81 \%$ (62) coverage.

Table 13: Type of QIPs Conduct by the Libraries

\begin{tabular}{|c|c|c|c|c|c|c|c|c|}
\hline Type of QIPs & $\begin{array}{c}\mathbf{U L} \\
(\mathrm{N}=\mathbf{3})\end{array}$ & $\begin{array}{c}\text { PL } \\
(\mathbf{N}=1)\end{array}$ & $\begin{array}{c}\mathrm{DL} \\
(\mathrm{N}=2)\end{array}$ & $\begin{array}{c}\mathrm{AL} \\
(\mathrm{N}=17 \\
)\end{array}$ & $\begin{array}{c}C L \\
(\mathrm{~N}=31 \\
)\end{array}$ & $\begin{array}{c}\text { LA } \\
(\mathrm{N}=16 \\
)\end{array}$ & $\begin{array}{c}\mathbf{O} \\
(\mathrm{N}=7)\end{array}$ & $\begin{array}{c}\text { Overall } \\
\text { Coverage } \\
(\mathrm{N}=77) \\
\end{array}$ \\
\hline $\begin{array}{l}\text { Conducting Literacy } \\
\text { Session }\end{array}$ & $\begin{array}{c}3 \\
(100 \% \\
)\end{array}$ & $\begin{array}{c}1 \\
(100 \% \\
)\end{array}$ & $\begin{array}{c}2 \\
(100 \% \\
)\end{array}$ & $\begin{array}{c}17 \\
(100 \% \\
)\end{array}$ & $\begin{array}{c}31 \\
(100 \% \\
)\end{array}$ & $\begin{array}{c}16 \\
(100 \% \\
)\end{array}$ & $\begin{array}{c}7 \\
(100 \% \\
)\end{array}$ & $\begin{array}{c}77 \\
(100 \%)\end{array}$ \\
\hline $\begin{array}{l}\text { Conducting inter \& } \\
\text { intra - library } \\
\text { discussion session }\end{array}$ & $\begin{array}{c}3 \\
(100 \% \\
)\end{array}$ & $\begin{array}{c}1 \\
(100 \% \\
)\end{array}$ & $\begin{array}{c}2 \\
(100 \% \\
)\end{array}$ & $\begin{array}{c}17 \\
(100 \% \\
)\end{array}$ & $\begin{array}{c}31 \\
(100 \% \\
)\end{array}$ & $\begin{array}{c}16 \\
(100 \% \\
)\end{array}$ & $\begin{array}{c}7 \\
(100 \% \\
)\end{array}$ & $\begin{array}{c}77 \\
(100 \%)\end{array}$ \\
\hline $\begin{array}{l}\text { Conducting } \\
\text { National/International } \\
\text { Webinars }\end{array}$ & $\begin{array}{c}3 \\
(100 \% \\
)\end{array}$ & $\begin{array}{c}1 \\
(100 \% \\
\quad) \\
\end{array}$ & $\begin{array}{c}2 \\
(100 \% \\
\quad) \\
\end{array}$ & $\begin{array}{c}17 \\
(100 \% \\
) \\
\end{array}$ & $\begin{array}{c}28 \\
(90 \%)\end{array}$ & $\begin{array}{c}14 \\
(88 \%)\end{array}$ & $\begin{array}{c}7 \\
(100 \% \\
\quad) \\
\end{array}$ & $\begin{array}{c}72 \\
(94 \%)\end{array}$ \\
\hline $\begin{array}{l}\text { Conducting Hands-on } \\
\text { Workshops }\end{array}$ & $\begin{array}{c}3 \\
(100 \% \\
)\end{array}$ & $\begin{array}{c}1 \\
(100 \% \\
\quad)\end{array}$ & $\begin{array}{c}2 \\
(100 \% \\
)\end{array}$ & $\begin{array}{c}14 \\
(82 \%)\end{array}$ & $\begin{array}{c}25 \\
(81 \%)\end{array}$ & $\begin{array}{c}14 \\
(88 \%)\end{array}$ & $\begin{array}{c}3 \\
(43 \%)\end{array}$ & $\begin{array}{c}62 \\
(81 \%)\end{array}$ \\
\hline $\begin{array}{l}\text { Conducting Short } \\
\text { Term Training } \\
\text { Programme (STTP) }\end{array}$ & $\begin{array}{c}1 \\
(33 \%)\end{array}$ & $\begin{array}{c}0 \\
(0 \%)\end{array}$ & $\begin{array}{c}1 \\
(50 \%)\end{array}$ & $\begin{array}{c}9 \\
(53 \%)\end{array}$ & $\begin{array}{c}0 \\
(0 \%)\end{array}$ & $\begin{array}{c}12 \\
(75 \%)\end{array}$ & $\begin{array}{c}4 \\
(57 \%)\end{array}$ & $\begin{array}{c}27 \\
(35 \%)\end{array}$ \\
\hline Any other types & $\begin{array}{c}1 \\
(33 \%)\end{array}$ & $\begin{array}{c}0 \\
(0 \%) \\
\end{array}$ & $\begin{array}{c}1 \\
(50 \%)\end{array}$ & $\begin{array}{c}4 \\
(24 \%) \\
\end{array}$ & $\begin{array}{c}8 \\
(26 \%) \\
\end{array}$ & $\begin{array}{c}0 \\
(0 \%) \\
\end{array}$ & $\begin{array}{c}2 \\
(29 \%) \\
\end{array}$ & $\begin{array}{c}16 \\
(21 \%) \\
\end{array}$ \\
\hline
\end{tabular}

SQ 10: Which type of learning method you are using for your staff members? 
For conducting QIPs or Literacy programmes, selection of appropriate method or mode of learning is also essential. Following Table 14 listed some of the commonly used learning methods by the libraries for their staff members. According to the received responses, the Online learning method used by all of the respondents (77 or 100\%). It is closely followed by Communication with peers ( 73 or $95 \%$ ) and Attending a webinar or conference in the next (67 or $87 \%)$.

Table 14: Types of Learning Methods used by the Staff Members

\begin{tabular}{|c|c|c|c|c|c|c|c|c|}
\hline Used Learning Methods & $\begin{array}{c}\mathbf{U L} \\
(\mathbf{N}=\mathbf{3})\end{array}$ & $\begin{array}{c}\text { PL } \\
(\mathbf{N}=\mathbf{1})\end{array}$ & $\begin{array}{c}\mathrm{DL} \\
(\mathrm{N}=2)\end{array}$ & $\begin{array}{c}\text { AL } \\
(\mathbf{N}=17)\end{array}$ & $\begin{array}{c}\text { CL } \\
(\mathbf{N}=\mathbf{3 1})\end{array}$ & $\begin{array}{c}\text { LA } \\
(\mathrm{N}=16 \\
)\end{array}$ & $\begin{array}{c}\mathbf{O} \\
(\mathbf{N}=7)\end{array}$ & $\begin{array}{c}\text { Overall } \\
\text { Coverag } \\
\text { e } \\
(\mathrm{N}=77) \\
\end{array}$ \\
\hline Online Learning & $\left|\begin{array}{c}3 \\
(100 \%)\end{array}\right|$ & $\begin{array}{c}1 \\
(100 \% \\
\quad\end{array}$ & $\begin{array}{c}2 \\
(100 \%)\end{array}$ & $\begin{array}{c}17 \\
(100 \%)\end{array}$ & $\begin{array}{c}31 \\
(100 \%)\end{array}$ & $\begin{array}{c}16 \\
(100 \% \\
)\end{array}$ & $\begin{array}{c}7 \\
(100 \%)\end{array}$ & $\begin{array}{c}77 \\
(100 \%)\end{array}$ \\
\hline Communications with Peers & $\begin{array}{c}3 \\
(100 \%)\end{array}$ & $\begin{array}{c}1 \\
(100 \% \\
\quad\end{array}$ & $\begin{array}{c}2 \\
(100 \%)\end{array}$ & $\begin{array}{c}17 \\
(100 \%)\end{array}$ & $\begin{array}{c}28 \\
(90 \%)\end{array}$ & $\begin{array}{c}15 \\
(94 \%)\end{array}$ & $\begin{array}{c}7 \\
(100 \%)\end{array}$ & $\begin{array}{c}73 \\
(95 \%)\end{array}$ \\
\hline Attending a webinar/conference & $\left|\begin{array}{c}3 \\
(100 \%)\end{array}\right|$ & $\begin{array}{c}1 \\
(100 \% \\
\end{array}$ & $\begin{array}{c}2 \\
(100 \%)\end{array}$ & $\begin{array}{c}17 \\
(100 \%)\end{array}$ & $\begin{array}{c}30 \\
(97 \%)\end{array}$ & $\begin{array}{c}10 \\
(63 \%)\end{array}$ & $\begin{array}{c}4 \\
(57 \%)\end{array}$ & $\begin{array}{c}67 \\
(87 \%)\end{array}$ \\
\hline Enroll free online courses & $\left|\begin{array}{c}3 \\
(100 \%)\end{array}\right|$ & $\begin{array}{c}1 \\
(100 \% \\
)\end{array}$ & $\begin{array}{c}2 \\
(100 \%)\end{array}$ & $\begin{array}{c}17 \\
(100 \%)\end{array}$ & $\begin{array}{c}22 \\
(71 \%)\end{array}$ & $\begin{array}{c}8 \\
(50 \%)\end{array}$ & $\begin{array}{c}0 \\
(0 \%)\end{array}$ & $\begin{array}{c}53 \\
(69 \%)\end{array}$ \\
\hline $\begin{array}{l}\text { Trough CAS Services (provided } \\
\text { by library) }\end{array}$ & $\begin{array}{c}3 \\
(100 \%)\end{array}$ & $\begin{array}{c}0 \\
(0 \%) \\
\end{array}$ & $\begin{array}{c}2 \\
(100 \%) \\
\end{array}$ & $\begin{array}{c}14 \\
(82 \%) \\
\end{array}$ & $\begin{array}{c}14 \\
(45 \%) \\
\end{array}$ & $\begin{array}{c}11 \\
(69 \%) \\
\end{array}$ & $\begin{array}{c}2 \\
(29 \%) \\
\end{array}$ & $\begin{array}{c}46 \\
(60 \%) \\
\end{array}$ \\
\hline Any other types & $\left|\begin{array}{c}3 \\
(100 \%)\end{array}\right|$ & $\begin{array}{c}1 \\
(100 \% \\
)\end{array}$ & $\begin{array}{c}2 \\
(100 \%)\end{array}$ & $\begin{array}{c}12 \\
(71 \%)\end{array}$ & $\begin{array}{c}10 \\
(32 \%)\end{array}$ & $\begin{array}{c}3 \\
(19 \%)\end{array}$ & $\begin{array}{c}0 \\
(0 \%)\end{array}$ & $\begin{array}{c}31 \\
(40 \%)\end{array}$ \\
\hline
\end{tabular}

SQ 11: Rate the efficiency of your available staff in disseminating information through online mode...

SQ 11 try to figure out the overall efficiency of the available library staffs of the respondents' libraries to disseminate information in online mode. The Overall coverage of Table 15 reveals that the majority of the respondent selects the Excellent, Very Good \& Good portion of the 5 points Likert scale with 39 (38\%), 29 (29\%) \& 24 (24\%) coverage, respectively.

Table 15: Efficiency of Available Library Staff in Online Information Dissemination

\begin{tabular}{|l|c|c|c|c|c|c|}
\hline Designation & Excellent & $\begin{array}{c}\text { Very } \\
\text { Good }\end{array}$ & Good & Fair & Poor & Total \\
\hline $\begin{array}{l}\text { University } \\
\text { Librarian }\end{array}$ & $\begin{array}{c}2 \\
(67 \%)\end{array}$ & $\begin{array}{c}1 \\
(33 \%)\end{array}$ & $\begin{array}{c}0 \\
(0 \%)\end{array}$ & $\begin{array}{c}0 \\
(0 \%)\end{array}$ & $\begin{array}{c}0 \\
(0 \%)\end{array}$ & $\begin{array}{c}\mathbf{3} \\
\mathbf{1 0 0 \%} \\
)\end{array}$ \\
\hline Public Librarian & 1 & $\begin{array}{c}2 \\
(25 \%)\end{array}$ & $\begin{array}{c}1 \\
(50 \%)\end{array}$ & $\begin{array}{c}0 \\
(25 \%)\end{array}$ & $\begin{array}{c}0 \\
(0 \%)\end{array}$ & $\begin{array}{c}\mathbf{4} \\
(\mathbf{1 0 0 \%} \\
)\end{array}$ \\
\hline
\end{tabular}




\begin{tabular}{|c|c|c|c|c|c|c|}
\hline Deputy Librarian & $\begin{array}{c}0 \\
(0 \%)\end{array}$ & $\begin{array}{c}2 \\
(100 \%)\end{array}$ & $\begin{array}{c}0 \\
(0 \%)\end{array}$ & $\begin{array}{c}0 \\
(0 \%)\end{array}$ & $\begin{array}{c}0 \\
(0 \%)\end{array}$ & $\begin{array}{c}2 \\
(100 \% \\
\quad)\end{array}$ \\
\hline $\begin{array}{l}\text { Assistant } \\
\text { Librarian }\end{array}$ & $\begin{array}{c}10 \\
(43 \%)\end{array}$ & $\begin{array}{c}4 \\
(17 \%)\end{array}$ & $\begin{array}{c}6 \\
(26 \%)\end{array}$ & $\begin{array}{c}3 \\
(13 \%)\end{array}$ & $\begin{array}{c}0 \\
(0 \%)\end{array}$ & $\begin{array}{c}23 \\
(100 \% \\
)\end{array}$ \\
\hline College Librarian & $\begin{array}{c}19 \\
(48 \%)\end{array}$ & $\begin{array}{c}11 \\
(28 \%)\end{array}$ & $\begin{array}{c}8 \\
(20 \%)\end{array}$ & $\begin{array}{c}0 \\
(0 \%)\end{array}$ & $\begin{array}{c}2 \\
(5 \%)\end{array}$ & $\begin{array}{c}40 \\
(100 \% \\
)\end{array}$ \\
\hline Library Assistant & $\begin{array}{c}7 \\
(39 \%)\end{array}$ & $\begin{array}{c}2 \\
(11 \%)\end{array}$ & $\begin{array}{c}6 \\
(33 \%)\end{array}$ & $\begin{array}{c}3 \\
(17 \%)\end{array}$ & $\begin{array}{c}0 \\
(0 \%)\end{array}$ & $\begin{array}{c}18 \\
(100 \% \\
)\end{array}$ \\
\hline Others & $\begin{array}{c}0 \\
(0 \%)\end{array}$ & $\begin{array}{c}7 \\
(64 \%)\end{array}$ & $\begin{array}{c}3 \\
(27 \%)\end{array}$ & $\begin{array}{c}0 \\
(0 \%)\end{array}$ & $\begin{array}{c}1 \\
(9 \%)\end{array}$ & $\begin{array}{c}11 \\
(100 \% \\
)\end{array}$ \\
\hline $\begin{array}{l}\text { Overall Coverage } \\
(\mathrm{N}=101)\end{array}$ & $\begin{array}{c}39 \\
(38 \%)\end{array}$ & $\begin{array}{c}29 \\
(29 \%)\end{array}$ & $\begin{array}{c}24 \\
(24 \%)\end{array}$ & $\begin{array}{c}6 \\
(6 \%)\end{array}$ & $\begin{array}{c}3 \\
(3 \%)\end{array}$ & $\begin{array}{c}101 \\
(100 \% \\
)\end{array}$ \\
\hline
\end{tabular}

SQ 12: Rate the infrastructural availability of your library in disseminating information through online mode...

In sequence with SQ 11, SQ 12 examines the infrastructural availability of the respondents' libraries in terms of online dissemination of information. Table 16 exhibits that the majority of respondents (62 or 61\%) asserted that their library has adequate infrastructural equipment to provide online library services. Some respondents chose "Available" (25 or 25\%) and "Partially Available" (11 or 11\%), while very few committed to "Fairly Available" (3 or 3\%). Notably, none of the respondents selected the "Not available at all" option, implying at least a minimum infrastructure available in the sample respondents' libraries.

Table 16: Infrastructural Availability in the Users' Library

\begin{tabular}{|c|c|c|c|c|c|c|}
\hline Designation & $\begin{array}{c}\text { Fully } \\
\text { Availabl } \\
\text { e } \\
\end{array}$ & Available & $\begin{array}{l}\text { Partially } \\
\text { Available }\end{array}$ & $\begin{array}{c}\text { Fairly } \\
\text { Availabl } \\
\text { e } \\
\end{array}$ & $\begin{array}{c}\text { Not } \\
\text { Availabl } \\
\text { e at all }\end{array}$ & Total \\
\hline University Librarian & $\begin{array}{c}3 \\
(100 \%)\end{array}$ & $\begin{array}{c}0 \\
(0 \%)\end{array}$ & $\begin{array}{c}0 \\
(0 \%)\end{array}$ & $\begin{array}{c}0 \\
(0 \%)\end{array}$ & $\begin{array}{c}0 \\
(0 \%)\end{array}$ & $\begin{array}{c}3 \\
(100 \% \\
)\end{array}$ \\
\hline Public Librarian & $\begin{array}{c}3 \\
(75 \%)\end{array}$ & $\begin{array}{c}1 \\
(25 \%)\end{array}$ & $\begin{array}{c}0 \\
(0 \%)\end{array}$ & $\begin{array}{c}0 \\
(0 \%)\end{array}$ & $\begin{array}{c}0 \\
(0 \%)\end{array}$ & $\begin{array}{c}4 \\
(100 \% \\
)\end{array}$ \\
\hline Deputy Librarian & $\begin{array}{c}1 \\
(50 \%)\end{array}$ & $\begin{array}{c}1 \\
(50 \%)\end{array}$ & $\begin{array}{c}0 \\
(0 \%)\end{array}$ & $\begin{array}{c}0 \\
(0 \%)\end{array}$ & $\begin{array}{c}0 \\
(0 \%)\end{array}$ & $\begin{array}{c}2 \\
(100 \% \\
)\end{array}$ \\
\hline Assistant Librarian & $\begin{array}{c}18 \\
(78 \%)\end{array}$ & $\begin{array}{c}2 \\
(9 \%)\end{array}$ & $\begin{array}{c}3 \\
(13 \%)\end{array}$ & $\begin{array}{c}0 \\
(0 \%)\end{array}$ & $\begin{array}{c}0 \\
(0 \%)\end{array}$ & $\begin{array}{c}23 \\
(100 \% \\
)\end{array}$ \\
\hline College Librarian & $\begin{array}{c}16 \\
(40 \%)\end{array}$ & $\begin{array}{c}17 \\
(43 \%)\end{array}$ & $\begin{array}{c}5 \\
(13 \%)\end{array}$ & $\begin{array}{c}2 \\
(5 \%)\end{array}$ & $\begin{array}{c}0 \\
(0 \%)\end{array}$ & $\begin{array}{c}40 \\
(100 \% \\
)\end{array}$ \\
\hline
\end{tabular}




\begin{tabular}{|c|c|c|c|c|c|c|}
\hline Library Assistant & $\begin{array}{c}13 \\
(72 \%)\end{array}$ & $\begin{array}{c}1 \\
(6 \%)\end{array}$ & $\begin{array}{c}3 \\
(17 \%)\end{array}$ & $\begin{array}{c}1 \\
(6 \%)\end{array}$ & $\begin{array}{c}0 \\
(0 \%)\end{array}$ & $\begin{array}{c}18 \\
(100 \% \\
)\end{array}$ \\
\hline Others & $\begin{array}{c}8 \\
(73 \%)\end{array}$ & $\begin{array}{c}3 \\
(27 \%)\end{array}$ & $\begin{array}{c}0 \\
(0 \%)\end{array}$ & $\begin{array}{c}0 \\
(0 \%)\end{array}$ & $\begin{array}{c}0 \\
(0 \%)\end{array}$ & $\begin{array}{c}11 \\
(100 \% \\
)\end{array}$ \\
\hline $\begin{array}{l}\text { Overall Coverage } \\
(\mathrm{N}=101)\end{array}$ & $\begin{array}{c}62 \\
(61 \%)\end{array}$ & $\begin{array}{c}25 \\
(25 \%)\end{array}$ & $\begin{array}{c}11 \\
(11 \%)\end{array}$ & $\begin{array}{c}3 \\
(3 \%)\end{array}$ & $\begin{array}{c}\mathbf{0} \\
(\mathbf{0} \%)\end{array}$ & $\begin{array}{c}101 \\
(100 \% \\
)\end{array}$ \\
\hline
\end{tabular}

\section{KEY FINDINGS:}

1. Among the respondent, $95 \%$ (96) demanded that their library provide online library services to their patrons during a lockdown.
a) All of these 96 respondent choose E-mail Management is the most commonly provided service, and Social Sites in exact next (86 or $96 \%$ ).
b) A total of 73 responded identified in providing a Remote Access Facility to their library users during lockdown period.

- Amongst them, the major choice is DELNET consortium (59 or $81 \%$ ), closely followed by EDS \& WoS, both with 67\% (49) coverage.

2. Except only 1 respondent from the study sample, $99 \%$ (100) respondent positive about their library to provide online ask a librarian facility during the lockdown.
a) According to these 100 respondents, 47\% (47) answer within 1 hour to any query received from the user. While, respondents who responded within 5 minutes came in second (33 or $33 \%$ ).
b) Again, out of the five mentioned web tools Gmail or Blue-mail services and Telephonic interaction used by $100 \%$ (100) of the respondent.

3. Considering overall coverage of the respondent at maximum $(\mathrm{N}=500), \leq 5$ queries per day is common $\&$ in the majority (41.2\% or 206) number for every type of user.

4. Out of the 101 sample respondent, $76 \%$ (77) ensured that their library conducts such QIPs for their staffs.
a) Literacy session and inter \& intra - library discussion session have the highest significance (77 or $100 \%$ ) among all of the QIPs.
b) Furthermore, all of the respondents (77 or 100\%) choose Online learning method for their overall literacy.

5. The majority of the respondent selects the Excellent, Very Good \& Good while describing the efficiency of their available staff information through online mode.

6. Similarly, in the case of infrastructural availability the coverage lies between Fully Available, Available \& Partially Available.

Overall, the study result proved the right selection of study samples and the target libraries for data collection. In the case of providing online library services \& online ask a librarian facility, the target libraries score high, but a few lacks identified while conducting QIPs for their staff members.

\section{CONCLUSION}


Libraries are the best source of information for sharing knowledge, support teaching-learning and scientific research. The present study investigated the current situation of the Covid-19 lockdown, as well as the role of LIS professionals in spreading information for day to day work purposes, as well as in educating \& informing people. Now, due to a shift in the way information is disseminated, several staff members are revealing concerns about their abilities and competencies. Training \& instructional programme should be implemented at an institutional level to make them confident about this severe \& drastic change in work culture. Further, this study highlights the necessity to initiate different activities like learning programs, workshops, webinars through collaboration between faculty and librarians. Nonetheless, the overall findings of this survey show that, even in these hectic situations, libraries have acted wisely and evolved into a continual knowledge factory. Further, the study findings also reveal that libraries are developing as a new generation of knowledge centres capable of assisting our nation in settling into a "new normal" condition.

\section{REFERENCES}

Andrews, M., Areekal, B., Rajesh, K., Krishnan, J., Suryakala, R., Krishnan, B., Muraly, C., \& Santhosh, P. (2020). First confirmed case of COVID-19 infection in India: A case report. Indian Journal of Medical Research, 115(5), 490-492. https://doi.org/10.4103/ijmr.ijmr_2131_20

Balachandar, P., \& Ramesh, R. (2019). INFORMATION AND COMMUNICATION TECHNOLOGY (ICT) IN LIBRARY OPERATIONS RESOURCES AND SERVICES IN UNIVERSITY LIBRARIES. International Journal of Recent Academic Research, 1(8), 472-474. https://journalijrar.com/sites/default/files/issues-pdf/IJRAR0427.pdf

Brown, W. K. (2020, May 1). Libraries Adapt amid Crisis: Finding inspiration from library workers across the country. American Libraries Magazine. https://americanlibrariesmagazine.org/2020/05/01/libraries-adapt-amid-crisis/

Connell, R., Wallis, L., \& Comeaux, D. (2021). The Impact of COVID-19 on the Use of Academic Library Resources Resource. Information Technology \& Libraries. https://digitalcommons.lsu.edu/cgi/viewcontent.cgi?article=1101\&context=libraries_p ubs

Dadhe, P., \& Dubey, M. (2020). Library Services Provided During COVID-19 Pandemic: Content Analysis of Websites of Premier Technological Institutions of India. Library Philosophy and Practice (E-Journal). https://digitalcommons.unl.edu/libphilprac/4445

Fasae, J. K., Adekoya, C. O., \& Adegbilero-Iwari, I. (2020). Academic libraries' response to the COVID-19 pandemic in Nigeria. Library Hi Tech, ahead-of-print(ahead-of-print). https://doi.org/10.1108/lht-07-2020-0166

IFLA. (2020, October 13). IFLA -- COVID-19 and the Global Library Field. https://www.ifla.org/covid-19-and-libraries 
Ishtiaq, S., Sehar, N., \& Shahid, A. (2020). Information Dissemination during Covid-19 and Lockdown: The Role of University libraries of Sindh, Pakistan. Library Philosophy and Practice (E-Journal). https://digitalcommons.unl.edu/libphilprac/4280

Press Information Bureau. (2020, March 24). Government of India issues Orders prescribing lockdown for containment of COVID-19 Epidemic in the country. Ministry of Home Affairs, Government of India. https://pib.gov.in/PressReleseDetail.aspx?PRID=1607997

Rafiq, M., Batool, S. H., Ali, A. F., \& Ullah, M. (2021). University libraries response to COVID-19 pandemic: A developing country perspective. The Journal of Academic Librarianship, 47(1), 102280. https://doi.org/10.1016/j.acalib.2020.102280

University Grant Commission. (2020). UGC Guidelines on Examinations and Academic Calendar for the Universities in View of COVID-19 Pandemic and Subsequent Lockdown. https://www.ugc.ac.in/pdfnews/4276446_UGC-Guidelines-onExaminations-and-Academic-Calendar.pdf

World Health Organization. (2020a, January 21). Novel Coronavirus (2019-nCoV). https://www.who.int/docs/default-source/coronaviruse/situation-reports/20200121sitrep-1-2019-ncov.pdf?sfvrsn=20a99c10_4

World Health Organization. (2020b, March 16). WHO Director-General's opening remarks at the media briefing on COVID-19 - 16 March 2020. https://www.who.int/directorgeneral/speeches/detail/who-director-general-s-opening-remarks-at-the-mediabriefing-on-covid-19---16-march-2020 